\title{
PRECOCIDADE DE PARTO NA ESTAÇÃO DE PARIÇÃO SOBRE A EFICIÊNCIA PRODUTIVA DE VACAS PRIMÍPARAS AOS 24 MESES DE IDADE
}

\section{PRECOCITY OF CALVING SEASON IN THE PRODUCTIVE EFFICIENCY OF PRIMIPAROUS COWS AT 24 MONTHS OF AGE}

\author{
Eduardo Madeira Castilho ${ }^{1}$ \\ Ricardo Zambarda Vaz ${ }^{*}$ \\ Tiago Albandes Fernandes ${ }^{1}$ \\ Viviane Garcia Dias da Conceição ${ }^{1}$ \\ Olivio Bochi Brum²
}

1Universidade Federal de Pelotas, Pelotas, RS, Brasil.

2Universidade Integrada do Alto Uruguai e Missões, Campus Santiago, RS, Brasil.

*Autor para correspondência - rzvaz@terra.com.br

\section{Resumo}

Objetivou-se verificar o efeito de dois períodos de parto, na estação de parição (precoces: setembro;

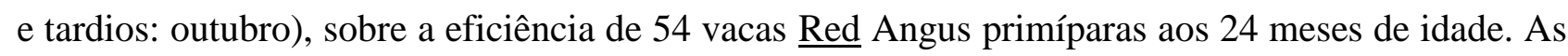
vacas foram mantidas em pastagem natural e cultivadas de Lotus corniculatus e Lolium multiflorum nos períodos pré e pós-parto, respectivamente. Utilizou-se inseminação artificial em tempo fixo, com dois protocolos, em intervalo de 30 dias. Realizaram-se diagnósticos de gestação 30 dias após cada protocolo. Bezerros de vacas paridas precocemente ganharam mais peso durante o aleitamento, sendo mais pesados ao desmame, quando comparados aos de vacas tardias, perfazendo maiores ganhos do conjunto vaca-bezerro durante a lactação $(0,721 \pm 0,030$ e $0,699 \pm 0,032 \mathrm{~kg})$. O desempenho reprodutivo foi superior paras as vacas paridas precocemente nas duas sincronizações e no índice de prenhez total (86,2 e 52\%). Vacas precoces na parição obtiveram maiores índices de produção de bezerros $(161,5 \pm 3,2$ e 92,5 $\pm 3,5 \mathrm{~kg})$, produção de bezerros $/ \mathrm{kg}$ de vaca ao desmame $(40,9 \pm 0,97 \mathrm{e}$ $23,7 \pm 1,04 \mathrm{~kg})$ e eficiência produtiva ao parto $(34,3 \pm 1,25$ e $20,8 \pm 1,35 \mathrm{~kg})$. Vacas paridas precocemente são mais eficientes na produção de $\mathrm{kg}$ de bezerros $/ \mathrm{kg}$ de vaca ao parto e ao desmame e, associando-se $\mathrm{kg}$ produzidos e prenhez subsequente, apresentam maior índice de produção de bezerros.

Palavras-chave: ganho de peso; índice de produção de bezerros; prenhez; Red Angus.

\footnotetext{
Abstract

The aim was to evaluate the effects of two calving periods, during the calving season (early: September; and late: October), in the efficiency of 54 Red Angus primiparous cows at 24 months of age. Over the pre-parturition and parturition periods, the cows were kept in natural and cultivated pastures of Lotus corniculatus and Lolium multiflorum, respectively. Fixed-time artificial
} 
insemination was used, with two protocols in a interval of 30-day. Pregnancy diagnosis was performed 30 days after each protocol. Calves from early calving cows presented larger weight daily gain, and were heavier at weaning when compared to later calving cows, resulting in higher cow-calf gains during the lactation $(0.721 \pm 0.030$ e $0.699 \pm 0.032 \mathrm{~kg})$. The reproductive performance was higher for the early calving cows in both synchronizations, and in the total pregnancy rate (86.2 and 52\%). Early calving cows had a higher calf production rate $(161.5 \pm 3.2$ e $92.5 \pm 3.5 \mathrm{~kg})$, calf $/ \mathrm{kg}$ of cow at weaning $(40.9 \pm 0.97$ e $23.7 \pm 1.04 \mathrm{~kg})$, and productive efficiency in the calving $(34.3 \pm 1.25$ e $20.8 \pm 1.35$ $\mathrm{kg}$ ). Early calving cows are more efficient in production of calves $\mathrm{kg} / \mathrm{cows} \mathrm{kg}$ in the calving and in the weaning, and when associated the kg produced and the subsequent pregnancy, presented a higher calf production rate.

Keywords: weight gain; index of calf production, pregnancy; Red Angus.

Recebido em: 28 de abril de 2017

Aceito em: 05 de outubro de 2017

\section{Introdução}

A pecuária de corte, nos últimos anos, passou por pouca evolução em seus índices de produtividade, demonstrando a real necessidade de manutenção da atividade ${ }^{(1)}$. Desta forma, a seleção de rebanhos mais produtivos e eficientes são fatores que contribuem para que produtores se mantenham na atividade $^{(2)}$. Quando se trata de produção e rentabilidade, pontos importantes como nutrição, reprodução, sanidade e melhoramento genético devem ter prioridade no sistema ${ }^{(3)}$.

A base da alimentação dos rebanhos de cria são as pastagens naturais. Estas sofrem oscilações e em determinadas épocas ofertam baixa quantidade e qualidade, causando deficiência nutricional para os animais ${ }^{(4)}$. Essa variação da disponibilidade de alimento compromete o peso ao nascer dos bezerros, além do ganho de peso até o desmame ${ }^{(5)}$.

A intensificação dos sistemas produtivos pode ser realizada com a redução da idade ao primeiro acasalamento de novilhas. Este fato irá diminuir as categorias de recria, devido ao aumento de fêmeas destinadas à reprodução, além de possibilitar um maior capital de giro dentro da propriedade ${ }^{(6)}$. Para isso, as exigências nutricionais destas novilhas são superiores, principalmente, quando estas se encontram prenhes aos 15 meses de idade, tornando-se primíparas aos 24 meses $^{(7)}$. Portanto, as primíparas devem ter adequados o ganho de peso durante a gestação e o escore de condição corporal ao $\operatorname{parto}^{(6)}$.

A repetição de prenhez em primíparas é um problema para os sistemas produtivos, independentemente do parto ser aos 24 ou 36 meses de idade ${ }^{(8)}$. A prenhez de primíparas pode ser melhorada, através da utilização de pastagens cultivadas de ciclo hibernal no pós-parto ${ }^{(9)}$, ajuste de carga animal ${ }^{(7)}$, utilização de desmame precoce ${ }^{(8)}$, utilização de suplementação estratégica no pré e pós-parto ${ }^{(6)}$. Além destes fatos, melhores escores de condição corporal e a idade são fatores a influenciar a prenhez de primíparas ${ }^{(10)}$.

O objetivo deste estudo foi verificar o efeito da data de parto (mês de parição) dentro da estação de nascimento sobre a eficiência produtiva e reprodutiva das vacas primíparas aos 24 meses de idade. 


\section{Material e Métodos}

O experimento foi realizado em uma propriedade particular, localizada no município de Arroio Grande, RS. Foram utilizadas 54 vacas Red Angus, primíparas aos 24 meses de idade, durante a sua primeira lactação. Estas vacas foram classificadas conforme a data de parto dentro da temporada de nascimento, em vacas precoces (partos durante o mês de setembro) ou tardias (partos durante o mês de outubro).

Durante o período pré-parto, as fêmeas foram mantidas em pastagem natural, com carga animal de $315 \mathrm{~kg} / \mathrm{ha}$. Após o parto, as vacas e seus bezerros foram identificados e pesados nas primeiras 24 horas, sendo conduzidos para uma área de pastagem cultivada, composta por cornichão (Lotus corniculatus) e azevém (Lolium multiflorum), com carga animal de $501 \mathrm{~kg} / \mathrm{ha}$. Ao desmame, os pares vacas e bezerros foram novamente pesados, sendo realizadas pesagens intermediárias, para o acompanhamento das cargas animais. As variações de pesos médios diários foram determinadas pela diferença entre as pesagens (quilogramas), divididas pelo período entre as mesmas (dias).

O desmame foi realizado quando os bezerros alcançaram idade média de 211 dias de idade (variação de 184 a 249 dias). Por ocasião das pesagens, foram realizadas as avaliações de escore de condição corporal das vacas, atribuindo-se valores dentro de uma escala de $1-5^{(11)}$.

O método de acasalamento utilizado na estação reprodutiva pós-parto foi o de inseminação artificial em tempo fixo (IATF), com dois protocolos em intervalo de 30 dias, antecedidos de avaliação ginecológica por ultrassonografia. Os diagnósticos de gestação foram realizados 30 dias após cada protocolo de IATF, sendo determinadas as prenhez por IATF e acumulada.

Para a produtividade do rebanho e eficiência das vacas em reprodução, foram considerados os pesos e ganhos totais de pesos corporais das vacas e bezerros do parto ao desmame, analisados individualmente ou no conjunto vaca-bezerro. A eficiência da produção de bezerros foi determinada através do índice de produção de bezerros ajustado ${ }^{(8)}$, de acordo com a taxa de prenhez em $\mathrm{kg}$ de bezerros desmamados por vaca mantida (peso dos bezerros ao desmame convencional*taxa de prenhez/100). As eficiências produtivas ao parto e ao desmame foram determinadas em função dos pesos dos bezerros ao desmame em relação ao das vacas nas mesmas datas ${ }^{(12)}$.

$\mathrm{O}$ delineamento experimental foi o inteiramente casualizado. Os resultados foram submetidos à análise de variância e ao teste F. O modelo matemático utilizado para as análises foi:

$Y i j=\mu+E i+S j+\Sigma i j$,

onde: Yij = variáveis dependentes; $\mu$ = média de todas as observações; $\mathrm{Ei}=$ efeito da i-ésima período de parto dentro da temporada de nascimentos, sendo $\mathrm{i}=1$ (metade inicial) e $\mathrm{i}=2$ (metade final); $\mathrm{Sj}=$ efeito j-ésimo sexo do bezerro como covariável, sendo $\mathrm{j}=1$ (macho) e j $=2$ (fêmea); $\Sigma \mathrm{ijk}=$ erro residual.

Os dados foram analisados utilizando-se o programa estatístico SAS ${ }^{(13)}$, adotando-se 5\% como nível de significância máxima. As análises foram realizadas com o auxílio do procedimento GLM. As médias foram comparadas pelo teste "F". A variável percentagem de prenhez nas duas épocas de 
nascimentos foi analisada pelo teste do Qui-Quadrado, ao nível de significância de 5\%.

\section{Resultados e Discussão}

As vacas, independentemente do mês de parição, não diferiram em pesos e condições corporais ao parto e ao desmame ( $\mathrm{P}>0,05$; Tabela 1$)$. Como os partos ocorreram nos meses de setembro e outubro, era esperado que houvesse uma diferença nestas variáveis, com maior peso para as vacas paridas mais tardiamente. Este maior peso das vacas mais tardias ao parto ocorre em função das mesmas serem beneficiadas por um maior período de pastagem na primavera, coincidindo com o terço final de gestação $^{(14)}$. Durante este período, ocorre uma melhora considerável da qualidade das mesmas ${ }^{(5)}$. No entanto, para vacas paridas precocemente, a qualidade das pastagens naturais logo após o parto é insuficiente para o aumento da condição corporal das vacas ${ }^{(15)}$. No entanto estas vacas possuem maior tempo entre o parto e o desmame, já tendo passado o pico da produção de leite, diminuindo o requerimento nutricional ${ }^{(15)}$.

Tabela 1. Médias e erros padrão para as características de desenvolvimento, reprodução e eficiência de vacas primíparas aos 24 meses de idade e seus bezerros, paridas em diferentes períodos dentro da estação de nascimentos

\begin{tabular}{|c|c|c|c|}
\hline Característica & $\mathbf{N}$ & Precoces & Tardias \\
\hline & & \multicolumn{2}{|c|}{ Pesos, $\mathrm{kg}$} \\
\hline Vaca ao parto & 54 & $385,2 \pm 5,6^{\mathrm{a}}$ & $376,4 \pm 6,0^{\mathrm{a}}$ \\
\hline Vaca ao desmame & 54 & $398,6 \pm 7,0^{\mathrm{a}}$ & $394,1 \pm 7,5^{\mathrm{a}}$ \\
\hline Bezerro ao nascimento & 54 & $48,4 \pm 1,5^{\mathrm{a}}$ & $47,7 \pm 1,6^{\mathrm{a}}$ \\
\hline \multirow[t]{2}{*}{ Bezerro ao desmame } & 54 & $187,3 \pm 4,3^{\mathrm{a}}$ & $177,7 \pm 4,6^{\mathrm{b}}$ \\
\hline & & \multicolumn{2}{|c|}{ Variações diárias de peso, $\mathrm{kg}$} \\
\hline Vaca do parto ao desmame & 54 & $0,063 \pm 0,028^{\mathrm{a}}$ & $0,083 \pm 0,030^{\mathrm{a}}$ \\
\hline Bezerro do nascimento ao desmame & 54 & $0,658 \pm 0,019^{\mathrm{a}}$ & $0,616 \pm 0,020^{b}$ \\
\hline \multirow[t]{2}{*}{ Conjunto vaca-bezerro durante a lactação } & 54 & $0,721 \pm 0,030^{\mathrm{a}}$ & $0,699 \pm 0,032^{\mathrm{b}}$ \\
\hline & & \multicolumn{2}{|c|}{ Condição corporal, pontos } \\
\hline Vaca ao parto & 54 & $3,2 \pm 0,04^{\mathrm{a}}$ & $3,1 \pm 0,04^{\mathrm{a}}$ \\
\hline Vaca ao desmame & 54 & $3,2 \pm 0,05^{\mathrm{a}}$ & $3,2 \pm 0,05^{\mathrm{a}}$ \\
\hline
\end{tabular}

a,b na mesma linha diferem $(P<0,05)$ pelo teste $\mathrm{F}$.

Por ocasião dos desmames, normalmente, espera-se maior peso das vacas paridas precocemente na temporada de nascimentos, fato não observado no presente estudo. O maior peso ao desmame das vacas paridas no início da temporada de parição é, em geral, devido ao maior intervalo de tempo do parto até o desmame. Com isso já haverá passado o pico de lactação e o balanço energético negativo do pós-parto estar diminuído pela mudança do hábito alimentar do bezerro, deixando de se alimentar unicamente do leite materno ${ }^{(15)}$.

Vacas paridas precocemente apresentaram do parto ao desmame variação diária de peso semelhante quando comparadas às vacas paridas tardiamente, com valores de 0,063 e 0,083 kg, respectivamente. 
Mesmo pelo fator negativo da amamentação e da presença do bezerro por maior período de tempo, como possíveis fatores inibidores da reprodução subsequente em vacas de corte ${ }^{(16)}$, os ganhos de pesos podem ser considerados bons, uma vez que as vacas eram primíparas aos 24 meses de idade, categoria que normalmente perde peso e condição corporal durante a lactação ${ }^{(17)}$. Para esta categoria, é fundamental o ganho de peso no período pós-parto, pois o mesmo está associado a melhores desempenhos reprodutivos ${ }^{(6)}$.

Animais jovens apresentam um maior peso relativo de tecidos que compõem os órgãos de alta intensidade metabólica, ao serem comparados com animais em idade adulta, fato que justifica a maior demanda energética por unidade de tamanho metabólico $\left(\mathrm{PV}^{0,75}\right)$ destes animais, sendo esta diferença reduzida conforme o peso vivo do animal cresce, ou aumentada conforme o peso vivo do animal decresce ${ }^{(17-20)}$. Em termos de partição e competição por nutrientes, a atividade ovariana é a função corporal de mais baixa prioridade, se comparada com as atividades vitais de manutenção das reservas corporais, crescimento, gestação e lactação ${ }^{(21-23)}$.

A repetição de prenhez das vacas paridas precocemente dentro da temporada de partos foi superior às paridas mais tardiamente $(\mathrm{P}<0,05$; Tabela 2$)$, independentemente da sequência de IATF, ou da prenhez total, com valores desta última de 86,2 e $52,0 \%$, respectivamente. Isso se deve, provavelmente, ao maior tempo pós-parto destas vacas, até o início do período reprodutivo subsequente. Isso porque o presente estudo ao ser realizado dentro de um sistema produtivo comercial, o desmame foi realizado em data única, independentemente da idade do bezerro. Maiores períodos de tempo do parto ao acasalamento são determinantes de maiores taxas de prenhez ${ }^{(24)}$. Partos precoces, com a melhora da qualidade e oferta das pastagens naturais, proporcionam melhores condições nutricionais, as quais favorecem às vacas, principalmente, durante o pico de lactação ${ }^{(25)}$, determinando melhores pesos de bezerros ao desmame ${ }^{(26)}$.

Primíparas paridas precocemente obtiveram maior prenhez na primeira sincronização para a realização da IATF quando comparadas às paridas tardiamente, com percentuais de 55,2 e 40,0\%, respectivamente. A prenhez no início do período reprodutivo é importante, pois se associa a maiores chances de sucesso nos períodos reprodutivos subsequentes, principalmente com a categoria de primíparas aos 24 meses, devido a sua diferenciação nas exigências nutricionais ${ }^{(27)}$.

Vaz et al. ${ }^{(2)}$, ao trabalharem com a mesma categoria, oriundas quando bezerras de diferentes idades de desmame e as avaliarem até a sua repetição de prenhez, verificaram que as maiores concentrações de estros nos períodos iniciais e intermediários do primeiro acasalamento quando novilhas repetiu-se no segundo período reprodutivo. Isso demonstra serem as novilhas que concebem no início do período reprodutivo e, consequentemente, parem no início da parição, as que apresentam maior taxa de prenhez quando primíparas. Maior concentração de partos no início da temporada de parição possibilita maior intervalo entre o parto e o fim do segundo período reprodutivo, determinantes do sucesso reprodutivo ${ }^{(28)}$. 
Tabela 2. Médias e erros padrão para eficiências reprodutivas e produtivas de vacas primíparas paridas aos 24 meses de idade, em diferentes períodos dentro das estações de nascimentos

\begin{tabular}{|c|c|c|c|}
\hline Característica & $\mathbf{N}$ & Precoces & Tardias \\
\hline & & \multicolumn{2}{|c|}{ Reprodução, \% } \\
\hline Taxa de prenhez primeira sincronização & 54 & $55,20^{\mathrm{A}}$ & $40,00^{\mathrm{B}}$ \\
\hline Taxa de prenhez segunda sincronização & 54 & $31,00^{\mathrm{A}}$ & $12,00^{\mathrm{B}}$ \\
\hline \multirow[t]{2}{*}{ Taxa de prenhez total } & 54 & $86,20^{\mathrm{A}}$ & $52,00^{\mathrm{B}}$ \\
\hline & & \multicolumn{2}{|c|}{ Pesos, $\mathrm{kg}$} \\
\hline Conjunto vaca-bezerro ao parto & 54 & $433,7 \pm 5,7^{\mathrm{a}}$ & $424,0 \pm 6,2^{\mathrm{a}}$ \\
\hline \multirow[t]{2}{*}{ Conjunto vaca-bezerro ao desmame } & 54 & $585,9 \pm 8,0^{\mathrm{a}}$ & $571,8 \pm 8,7^{\mathrm{a}}$ \\
\hline & & \multicolumn{2}{|c|}{ Eficiências, $\mathrm{kg}$} \\
\hline Índice de produção de bezerros ${ }^{1}$ & 54 & $161,5 \pm 3,2^{\mathrm{a}}$ & $92,5 \pm 3,5^{b}$ \\
\hline Produção de bezerros $/ \mathrm{kg} \mathrm{vaca}{ }^{2}$ & 54 & $40,9 \pm 0,97^{\mathrm{a}}$ & $23,7 \pm 1,04^{b}$ \\
\hline Eficiência produtiva da vaca ao parto ${ }^{3}$ & 54 & $12,28 \pm 0,45^{\mathrm{a}}$ & $12,17 \pm 0,49^{\mathrm{a}}$ \\
\hline Eficiência produtiva da vaca ao desmame ${ }^{4}$ & 54 & $47,4 \pm 1,35^{\mathrm{a}}$ & $45,6 \pm 1,46^{\mathrm{a}}$ \\
\hline \multirow[t]{2}{*}{ Eficiência de produção ao parto 5} & 54 & $34,3 \pm 1,25^{\mathrm{a}}$ & $20,8 \pm 1,35^{\mathrm{b}}$ \\
\hline & \multicolumn{3}{|c|}{ Variações totais de peso, $\mathrm{kg}$} \\
\hline Bezerro durante a amamentação & 54 & $138,9 \pm 4,25^{\mathrm{a}}$ & $130,1 \pm 4,5^{\mathrm{a}}$ \\
\hline Vaca na lactação & 54 & $13,3 \pm 5,6^{\mathrm{a}}$ & $17,7 \pm 6,1^{\mathrm{a}}$ \\
\hline Conjunto vaca-bezerro na amamentação & 54 & $152,2 \pm 6,3^{\mathrm{a}}$ & $147,7 \pm 6,8^{\mathrm{a}}$ \\
\hline
\end{tabular}

Os pesos do conjunto vaca-bezerro não diferiram ao parto e ao desmame, bem como nas variações diárias e totais de peso $(\mathrm{P}>0,05)$ entre as épocas de parição dentro da temporada de partos. Esse fato se deve, provavelmente, ao manejo despendido à categoria no pré-parto e à alimentação no pós-parto, de qualidade suficiente para a categoria em estudo. A similaridade de pesos das vacas e bezerros ao parto e ao desmame determinaram eficiências produtivas de quilogramas de bezerros ao desmame, em relação aos pesos das vacas ao parto e ao desmame, similares entre as épocas de parição dentro da temporada $(\mathrm{P}>0,05)$.

No entanto, ao associar pesos dos bezerros ajustados para a prenhez das vacas, verifica-se uma superioridade das vacas precoces no parto, quando comparadas às tardias. Vacas paridas no início da temporada de parição, independentemente da forma da avaliação, considerando peso ao desmame dos bezerros e a taxa de prenhez, foram em média 74,59 e 72,57\% superiores às paridas tardiamente, sem ou com ajuste ao peso de desmame das vacas, respectivamente. Estes indicadores, embora sejam prováveis produções no ano subsequente por vaca mantida, possuem um valor no que se refere à manutenção dos animais no rebanho de cria. Pensando em eficiência, a vaca para ser considerada 
produtiva deve produzir um bezerro por ano ${ }^{(2)}$.

No caso da idade de acasalamento das novilhas, a redução da mesma é fundamental para a retirada de categorias ineficientes do sistema produtivo, bem como é determinante de aumento das taxas de desfrute dos rebanhos ${ }^{(6)}$. Porém, de nada vale esta prenhez precoce das novilhas se as mesmas não repetirem cria, sendo perdida toda a alimentação diferenciada para acelerar o processo de puberdade e acasalamento ${ }^{(27)}$, bem como sendo descartados por infertilidade animais geneticamente superiores em rebanhos que trabalham com melhoramento genético ${ }^{(29)}$. Isso é determinado, provavelmente, pelo maior tempo de recuperação das vacas, com maiores pesos, condições corporais e, consequentemente, melhor resposta reprodutiva ${ }^{(9)}$.

\section{Conclusões}

Bezerros nascidos precocemente dentro da estação de parição são mais pesados ao desmame do que os filhos de vacas paridas na metade final da estação.

Parições precoces são determinantes de maiores taxas de prenhez no período reprodutivo subsequente.

Vacas paridas precocemente são mais eficientes na produção de quilogramas de bezerro por quilograma de vaca ao parto e ao desmame, possuindo maior índice de produção de bezerros.

\section{Referências}

1. ANUALPEC. Anuário da Pecuária Brasileira. São Paulo: FNP; 2016. 271 p. Portuguese.

2. Vaz RZ, Lobato JFP, Restle J. Influence of weaning age on the reproductive efficiency of primiparous cows. Revista Brasileira de Zootecnia [Internet]. 2010 [cited 2017 Nov 6];39(2):299-307. Available from: http://dx.doi.org/10.1590/S1516-35982010000200011. English.

3. Silva FL, Fraga AB, Espíndola Filho AM, Pedrosa AC. Desempenho de bovinos no Estado de Alagoas. Revista Brasileira de Saúde e Produção Animal [Internet]. 2008 [cited 2017 Nov 6];9(2):219-230. Available from: http://revistas.ufba.br/index.php/rbspa/article/viewArticle/982. Portuguese.

4. Vieira DH, Medeiros LFD, Barbosa CG, Rodrigues VC, Mello MRB, Oliveira JP. Efeitos não genéticos sobre as características reprodutivas de fêmeas da raça Nelore. II - Idade à primeira parição e intervalo de parto. Revista Brasileira de Medicina Veterinária [Internet]. 2010 [cited 2017 Nov 6];32(2):79-88. Available from: http://www.rbmv.com.br/pdf_artigos/03-08-2010_22-04RBMV003.pdf. Portuguese.

5. Corrêa MBB, Dionello NJL, Cardoso FF. Influência ambiental sobre características de desempenho prédesmama de bovinos Devon no Rio Grande do Sul. Revista Brasileira de Zootecnia [Internet]. 2006 [cited 2017 Nov 6];35(3S):1005-1011. Available from: http://dx.doi.org/10.1590/S1516-35982006000400010. Portuguese.

6. Pilau A, Lobato JFP. Suplementação energética pré-acasalamento aos 13/15 meses de idade para novilhas de corte: desenvolvimento e desempenho reprodutivo. Revista Brasileira de Zootecnia [Internet]. 2009 [cited 2017 Nov 6];38(12):2482-2489. Available from: http://dx.doi.org/10.1590/S1516-35982009001200026. Portuguese. 
7. Pötter BAA, Lobato JFP. Efeitos de carga animal, pastagem melhorada e idade de desmame no comportamento reprodutivo de vacas primíparas. Revista Brasileira de Zootecnia [Internet]. 2004 [cited 2017 Nov 6];33(1):192-202. Available from: http://dx.doi.org/10.1590/S1516-35982004000100023. Portuguese.

8. Vaz RZ, Lobato JFP. Effects of the weaning age of calves on somatic development and on reproductive performance of beef cows. Revista Brasileira de Zootecnia [Internet]. 2010 [cited 2017 Nov 6];39(5):10581067. Available from: http://dx.doi.org/10.1590/S1516-35982010000500016. English.

9. Lobato JFP, Menegaz AL, Pereira ACG. Pre and post-calving forage systems and reproductive performance of primiparous cows. Revista Brasileira de Zootecnia [Internet]. 2010 [cited 2017 Nov 6];39(9):2081-2090. Available from: http://dx.doi.org/10.1590/S1516-35982010000900029. English.

10. Vieira A, Lobato JFP, Torres Junior RAA, Cezar IM, Corrêa ES. Fatores determinantes do desempenho reprodutivo de vacas Nelore na região dos Cerrados do Brasil Central. Revista Brasileira de Zootecnia [Internet]. 2005 [cited 2017 Nov 6];34(6):2408-2416. Available from: http://dx.doi.org/10.1590/S151635982005000700029. Portuguese.

11. Marques PR. Planejamento e gestão do sistema de cria. In: Menegassi SRO, Canellas LC, Marques PR, Moojen FG, Azevedo EVT, Evangelista GT, et al. Manejo de sistemas de cria em pecuária de corte. Guaíba: Agrolivros; 2013. p. 141-155. Portuguese.

12. Ribeiro ELA, Restle, J, Rocha MA, Mizubuti IY, Silva LDF. Eficiência produtiva em vacas primíparas das raças Aberdeen Angus e Charolês. Revista Brasileira de Zootecnia [Internet]. 2001 [cited 2017 Nov 6];30(1):125-132. Available from: http://dx.doi.org/10.1590/S1516-35982001000100019. Portuguese.

13. SAS Institute. Statistical Analysis System: user guide [CD-ROM]. Version 6.08. Cary: SAS Insitute Inc.; 1997. English.

14. Knapik K, Kozicki LE, Segui MS, Pereira A, Breda JCS, Pimpão CT, et al. Desempenhos reprodutivos de bovinos de corte da raça Brangus e sua relação com os meses da estação de parturição no Sul brasileiro. Revista Acadêmica, Ciências Agrárias Ambientais [Internet]. 2011 [cited 2017 Nov 6];9(3):263-269. Available from: http://www2.pucpr.br/reol/pb/index.php/academica?dd1=5992\&dd99=view\&dd98=pb. Portuguese.

15. Condorelli EMF, Salomoni E, Oliveira NM. Desenvolvimento ponderal de novilhas Brangus-Ibagé nascidas em diferentes épocas do período inverno-primavera. Revista Brasileira de Agrociência [Internet]. 2000 [cited 2017 Nov 6];6(1):59-65. Available from: https://periodicos.ufpel.edu.br/ojs2/index.php/CAST/article/view/313. Portuguese.

16. Montiel F, Ahuja C. Body condition and suckling as factors influencing the duration of postpartum anestrus in cattle: a review. Animal Reproduction Science [Internet]. 2005 [cited 2017 Nov 6];85(1):1-26. Available from: http://dx.doi.org/10.1016/j.anireprosci.2003.11.001. English.

17. Di Marco ON, Barcellos JOJ, Da Costa EC. Crescimento de bovinos de corte. Porto Alegre: UFRGS; 2007. 278 p. Portuguese.

18. Smith NE, Baldwin RL. Effects of Breed, Pregnancy, and Lactation on Weight of Organs and Tissues in Dairy Cattle. Journal of Dairy Science [Internet]. 1974 [cited 2017 Nov 6];57(9):1055-1060. Available from: http://dx.doi.org/10.3168/jds.S0022-0302(74)85008-3. English.

19. Baldwin BR, Forsberg NE, Hu CY. Potential for Altering Energy Partition in the Lactating Cow. Journal of Dairy Science [Internet]. 1985 [cited 2017 Nov 6];68(12):3394-3402. Available from: http://dx.doi.org/10.3168/jds.S0022-0302(85)81252-2. English.

20. Ferrel CL. Contribution of visceral organs to animal energy expenditures. Journal of Animal Science [Internet]. 1988 [cited 2017 Nov 6];66(3):23-34. Available from: https://www.researchgate.net/publication/267197630_Contribution_of_visceral_organs_to_animal_energy_e 
xpenditures. English.

21. Dunn TG. Relationship of nutrition to successful embryo transplantation. Theriogenology [Internet]. 1980 [cited 2017 Nov 6];13(1):27-39. Available from: http://dx.doi.org/10.1016/0093-691X(80)90012-6. English.

22. Short RE, Bellows RA, Staigmiller RB, Berardinelli JG, Custer EE. Physiological mechanisms controlling anestrus and infertility in postpartum beef cattle. Journal of Animal Science [Internet]. 1990 [cited 2017 Nov 6];68(5):799-816.

Available

from:

https://www.animalsciencepublications.org/publications/jas/articles/68/3/799. English.

23. Schillo KK. Effects of dietary energy on control of luteinizing hormone secretion in cattle and sheep. Journal of Animal Science [Internet]. 1992 [cited 2017 Nov 6];70(4):1271-1282. Available from: https://www.animalsciencepublications.org/publications/jas/articles/70/4/1271. English.

24. Viu MAO, Brasil IG, Lopes DT, Gambarini ML, Ferraz HT, Oliveira Filho BD, et al. Fertilidade real e intervalo de partos de vacas nelore $\mathrm{PO}$ sob manejo extensivo e sem estação de monta na região centro oeste do Brasil. Bioscience Journal [Internet]. 2008 [cited 2017 Nov 6];24(1):104-111. Available from: http://www.seer.ufu.br/index.php/biosciencejournal/article/view/6760. Portuguese.

25. Pellegrini CB, Lopes LFD. Comportamento reprodutivo de vacas de corte manejadas em pastagem natural com duas cargas animais e duas idades à desmama. Revista Brasileira de Zootecnia [Internet]. 2011 [cited 2017 Nov 6];40(11):2606-2612. Available from: http://dx.doi.org/10.1590/S1516-35982011001100044. Portuguese.

26. Bagley CP, Carpenter JC Jr, Feazer JI, Hembry FG, Huffman DC, Koonce KL. Influence of calving season and stocking rate on beef cow-calf productivity. Journal of Animal Science [Internet]. 1987 [cited 2017 Nov 6];64(3):687-694. Available from: https://www.animalsciencepublications.org/publications/jas/abstracts/64/3/JAN0640030687. English.

27. Vaz RZ, Restle J, Pacheco PS, Vaz FN, Pascoal LL, Vaz MB. Ganho de peso pré e pós-desmame no desempenho reprodutivo de novilhas de corte aos quatorze meses de idade. Ciência Animal Brasileira [Internet]. 2012 [cited 2017 Nov 6];13(2):272-281. Available from: https://www.revistas.ufg.br/vet/article/view/17527. Portuguese.

28. Osoro K, Wright IA. The effect of body condition, live weight, breed, age, calf performance, and calving date on reproductive performance of spring-calving beef cows. Journal of Animal Science [Internet]. 1992 [cited 2017 Nov 6];70(6):1661-1666. Available from: https://www.animalsciencepublications.org/publications/jas/abstracts/70/6/1661?search-result=1. English.

29. Funston RN, Larson DM. Heifer development systems: Dry-lot feeding compared with grazing dormant winter forage. Journal Animal Science [Internet]. 2011 [cited 2017 Nov 6];89(5):1595-1602. Available from: https://www.animalsciencepublications.org/publications/jas/abstracts/89/5/1595? search-result=1. English. 